\title{
An Agent Model for Managing Distributed Software Resources in Grid Environment
}

\author{
Jingbo Ding and Weiqin Tong \\ School of Computer Engineering and Science, \\ Shanghai University, Shanghai 200072, China \\ dingjingbo@etang.com
}

\begin{abstract}
Grid technologies enable large-scale sharing of many types of resources, among which software resources are a vital part. Abundant software resources exist in geographically distributed hosts in grid environment. The characteristic "distributed" makes the discovery, characterization, and monitoring of them challenging due to the considerable diversity, large numbers, dynamic behavior, and geographical distribution of the software entities. Thus, we put forward here an agent model, which is built on grid technologies, to manage the software resources in grid environment. The agent can store, retrieve and manage the information of software resources through a resource directory. Our model also provides a visualizing uniform interface by which user can easily find software resources in grid and utilize them. To sum up, this agent is a middleware between end user and software resources.
\end{abstract}

\section{Introduction}

Grid computing technologies enable widespread sharing and coordinated use of networked resources, and make remote utilization of distributed software resources possible $[4,5,10,15,1]$. But users often feel inconvenient when using these distributed resources in grid environment. For this reason, it is necessary to design an agent model, which is designated to support the initial discovery and ongoing utilization of the existence and characteristics of software resources.

The goal of our model is to provide a software resource pool, which can collect the information of software resources, and to provide an interface and a mechanism by which end users can discover and then utilize appropriate software entities interested.

The "distributed" characteristic of software resources in grid environment can be viewed as Fig. 1. All the software entities are geographically distributed and owned by Internet hosts. As Fig. 2 shows, we can regard them together as a resource tree. A virtual grid root is defined at the top of tree for convenience of undertaking. Software entities belong to hosts, which are a part of domain or connected to Internet directly.

Our agent model provides a software resources pool, which can be seen as a logical information tree. Fig. 3 describes an example. In this tree, all the software resources are managed centrally and classified in order to provide a uniform view to users. This mechanism makes managing software resources in grid computation environment more efficient and provides an encapsulated, uniform interface for user. For example, users can use software resources in grid without knowing what they are 
and where they exist. By using this agent, user can find adequate information of software resources conveniently.

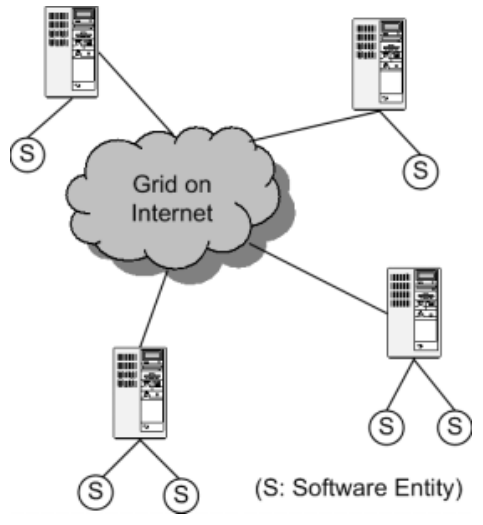

Fig. 1. Distributed software resources in grid environment

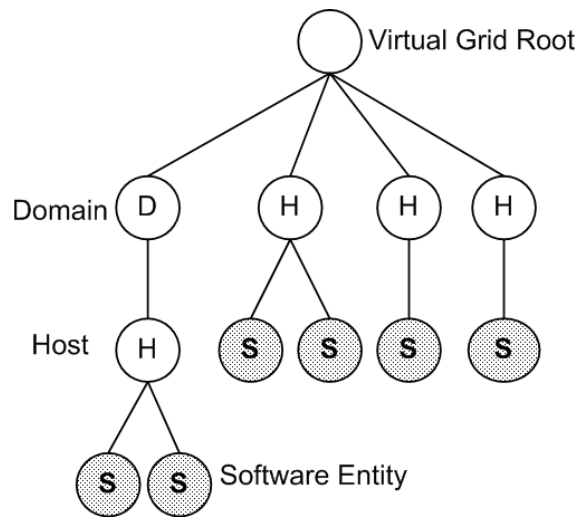

Fig. 2. A sample of resource tree

Let us compare the two trees. The former "resource tree" reflects the original characteristic of software resources - distributed; while the later "logical information tree", which satisfies users' needs, does conceal this characteristic. The main goal of our agent is to provide a logical view of software resources to end-users.

In a word, our agent for managing distributed software resources is a middleware between users and software resources that can: 1) for software resources, construct a centralized resource pool to store information of software resources and some maintenance tools to manage it; 2) for users, provide a visualizing uniform interface by with users can easily find and utilize them in grid environment.

The architecture of our model consists of four basic elements:

- Resource directory. This is a database that contains the information of software resources. In our model, we use LDAP (Lightweight Directory Access Protocol) [6, 2, 3] as its based protocol.

- Registry tool for software resources. Software resources should be initially registered to a centralized resource directory, which stores the information of software resources, to enable themselves to be discovered. Registry tool provides operation interface by which software resources can be registered in the directory. But software resources in grid environment are highly dynamic. To adapt for this characteristic, the agent uses a corresponding mechanism that updates the resource directory when some changes happen in software resources.

- Discovery tool for users. The main function of this tool is to accept querying request from users, search information in directory, and reply results back. Another purpose of this tool is to make users able to maintain the resource directory, such as add, delete, and modify information in it.

- Web interface. To provide a platform-irrespective operation and a friendly interface, web interface adopts XML technologies [12] to produce uniform 
data format. An interpreter converts the data between web interface and agent kernel commands, such as "register" and "query".

The rest of this paper is structured as follows. In the next section, we briefly review the background of grid computation and some related work. In subsequent sections we first outline our architecture and then describe our agent model in detail: data model, registry tool, and discovering process. In Sect. 7 and Sect. 8, we present our trace of OGSA proposal and give a famous related work. We summarize the paper and conclude with a discussion of some future directions in Sect. 9.

\section{Background}

\subsection{Grid and Globus Toolkit}

A computational grid is a hardware and software infrastructure that provides access to high-end computational capabilities. Grid computing is concerned with coordinated resource sharing and problem solving in dynamic, multi-institutional virtual organizations $[5,10,15]$. The key concept is the ability to negotiate resource-sharing arrangements among a set of participating parties (providers and consumers) and then to use the resulting resource pool for some purpose. The Globus Project provides software tools that make it easier to build computational grids and grid-based applications. These tools are collectively called the Globus Toolkit, which provides three elements (Resource Management, Information Services, and Data Management) necessary for computing in a grid environment $[9,11]$.

The relationship between our agent model and Globus Toolkit is as following. Our agent model is based on Globus Toolkit. Parts of our agent work through the API of Globus Toolkit.

\subsection{Directory, Directory Service, and Grid Information Services}

Directory. Directories are used to store and retrieve information [3, 6]. Thus, directories are similar to databases.

Directory Service. A Directory Service provides a directory that can be accessed via a network protocol. Often, directory services include mechanisms for replication and data distribution. An example of directory service is Domain Name System (DNS), which resolves the names of computers to appropriate addresses [2, 3].

LDAP. The abbreviation LDAP stands for Lightweight Directory Access Protocol. LDAP defines a standard directory protocol that includes the following features $[3,6]$ :

- A network protocol for accessing information in the directory.

- An information model defining the form and character of the information.

- A namespace defining how information is referenced and organized.

- An emerging distributed operation defining how data may be distributed and referenced (LDAP version 3 [6]).

- An extensible protocol.

- An extensible information model.

Information Services are utilities that provide information about grid resources, including the Monitoring and Discovery Service (MDS), which provides a 
configurable information provider component called a Grid Resource Information Service (GRIS) and a configurable aggregate directory component called a Grid Index Information Service (GIIS) [1, 7, 8, 9]. The MDS uses the LDAP as a uniform interface to such information. A variety of software components, ranging from commercial servers to open source code, can be used to implement the basic features of MDS: generating, publishing, storing, searching, querying, and displaying the data.

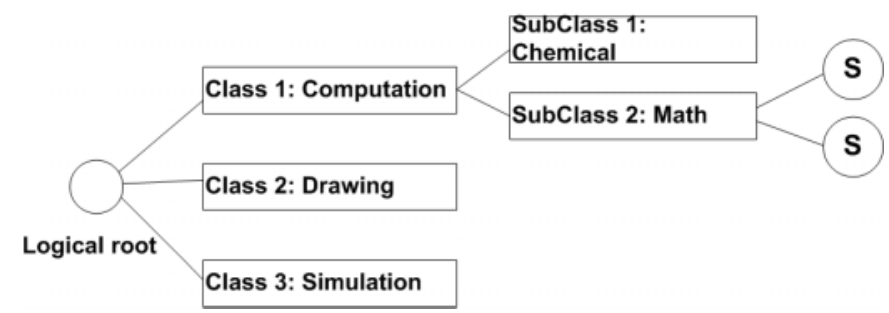

Fig. 3. A sample of logical view of resource tree

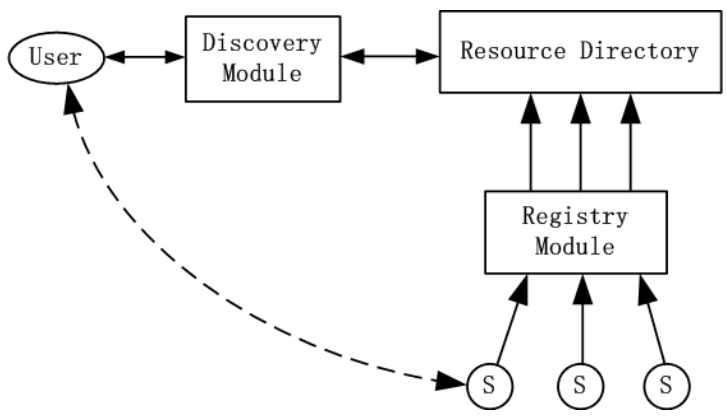

Fig. 4. Architecture overview

\section{Architecture Overview}

As described in Sect. 2, in Grid Information Services, with using the GRid Information Protocol (GRIP), users can query aggregate directory services to discover relevant entities, and/or query information providers to obtain information about individual entities. And description services are normally hosted by a grid entity directly, or by a front-end gateway serving the entity [1]. Now that software resource is a kind of resources, and it can be specified based on its information, we put forward such a motivation that utilizing the Grid Information Services to make software resources sharing possible.

The architecture of our agent model, which is described as Fig. 4, comprises three main parts - specialized aggregate resource directory, registry module, and discovery module. Software entities put their description information into resource directory through registry module. So directory stores gathered information of them. Using discovery module, users can query aggregate resource directory to discover relevant software entities and obtain information about individual entities. 
Grid Information Service defines two basic protocols, the GRid Information Protocol (GRIP) and the GRid Registration Protocol (GRRP), to build a directory service [1,9]. GRIP is used to access information about entities, while GRRP is used to notify aggregate directory services of the availability of this information [1]. We utilize these two protocols to realize our model, as we will describe in detail below.

Registry and discovery module are defined as services. In the process of registry, information of resources is collected in directory by registry module based on GRRP. Users use GRIP to query resources entities through discovery module. That is to say, registry service is used to register information of software resources, while discovery service is utilized to look up appropriate software entities for users.

\section{Data Model}

\subsection{Utilizing Data Model of LDAP}

In LDAP, the information model and namespace are based on entries. An entry is used to store attributes. An attribute has an associated type and can have one or more values. Each entry in the namespace has a distinguished name that allows easy identification $[3,6]$.

Entries are organized to form a hierarchical, tree-structured name space called a directory information tree (DIT). The distinguished name for an entry is constructed by specifying the entries on the path from the DIT root to the entry being named $[2,3$, 6].

The data model in our agent is organized by logical information tree of software resources (See Fig. 3). Each object is stored in the directory as an item. The attribute softwareclass indicates the class to which the resource belongs. Hence, the agent can classify software resources by their attributes. On the other hand, the data structure of our model accords with the LDAP data model. So we can use LDAP to communicate between registry tool and resource directory.

An example is given to illustrate data model of our agent model, where a distinguished name could be as following:

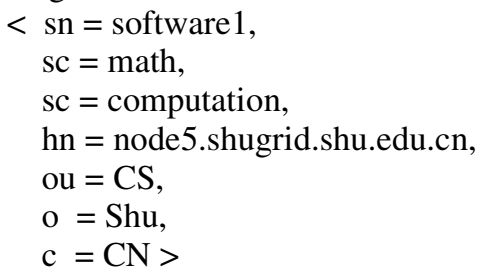

Like MDS, the components of the distinguished name are listed in little endian order, with the component corresponding to the root of the DIT [2]. Within a distinguished name, abbreviated attributes are typically used. In this example, the names of the distinguishing attributes are: software name $(\mathrm{SN})$, software class (SC), host name $(\mathrm{HN})$, organizational unit $(\mathrm{OU})$, organization $(\mathrm{O})$, and country $(\mathrm{C})$. Thus, a country entry is at the root of the DIT, while host entries are located beneath the organizational unit level of the DIT. So the software entities belonging to specified host can be located distinctively. 


\subsection{Specification of Software Resources Information}

Each object of software entities is stored in resource directory as an item. To search software resources and acquire adequate information, the attributes of each item should at least include: software name, class to which resource belongs, owner, host name and IP address of the host in which resource exists, local directory in the remote host, execution file name, software description and other information necessarily. A specialized MDS object class "GridSoftware" is created to contain these attributes. Thus, a software entity can be represented by data model of agent. For the example above, the directory should include an item such as:

dn: $\quad<$ sn=equation, $\quad \mathrm{sc}=$ math, $\quad \mathrm{sc}=$ computation, $\mathrm{hn}=$ node1. shugrid2.shu.edu.cn, ou=shugrid $2, \mathrm{o}=\mathrm{Shu}, \mathrm{c}=\mathrm{CN}>$

objectclass: GridSoftware

owner: $<\mathrm{cn}=$ Person $1, \mathrm{ou}=\mathrm{CS}, \mathrm{o}=\mathrm{Shu}, \mathrm{c}=\mathrm{CN}>$

softwareclass: math

softwareclass: computation

hostname: node5.shugrid.shu.edu.cn

IP: 202.120.113.199

localdir: /usr/local/mathtool

execution: math1

description: Description text of this entity

To make process of searching entities more efficient, an additional database is used to store information of software resources separately from DIT. It is used for speeding up searching process. Each item in this database is corresponding to the same entity in DIT. When users look for some entity in resource pool, additional database is searched first, and then the agent searches relevant entries in DIT.

\subsection{Information Directory}

Information Directory is a vital part of our agent model. A vital one of the three elements of our agent model (See Sect. 1) is information directory, which is used to store information of software resources. We now describe the realization of information directory.

Since we use LDAP as our based protocol, let us first review DIT used by MDS. As introduced in Sect. 2 and Sect. 4.1, the information model of LDAP are based on entries, which are organized to form a DIT. A subset of DIT defined in MDS, which is showed in Fig. 5, is typical for LDAP directory structures, looking similar to the organization used for multinational corporations [2].

As we described in Sect. 3.1, our motivation of designing this agent model is to utilize Grid Information Services in grid technologies to manage distributed software resources. In our agent, we merge the information of software resources into DIT. An additional quick database is used to speed up searching process. It stores information of software resources in DIT in particular. Each item in this database is corresponding to the same entity in DIT. When users look for some entity in resource pool, additional database is searched first, and then the agent searches relevant entries in 
DIT. The situation that information merged into DIT and the relationship between DIT and its additional quick database is showed in Fig. 6.

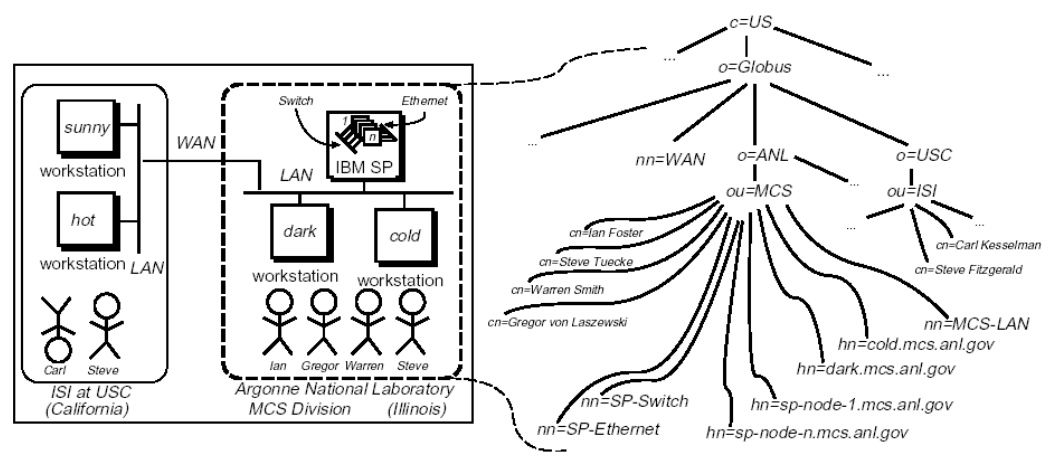

Fig. 5. A subset of DIT defined by MDS, showing the organizational nodes for Globus, ANL, and USC; the organizational units ISI and MCS, and people, hosts, and networks [2].

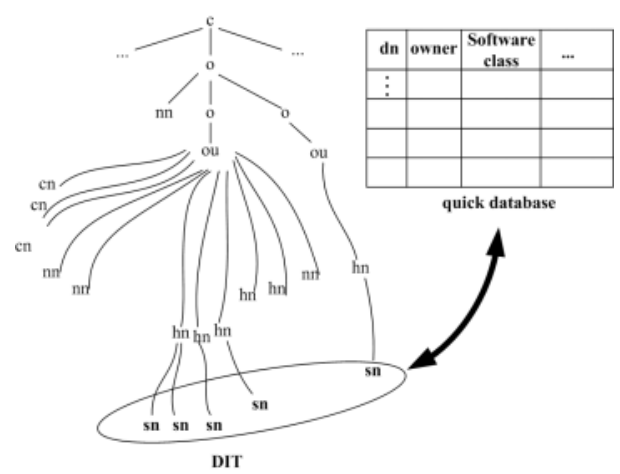

Fig. 6. Using quick database in the information directory to speed up searching process.

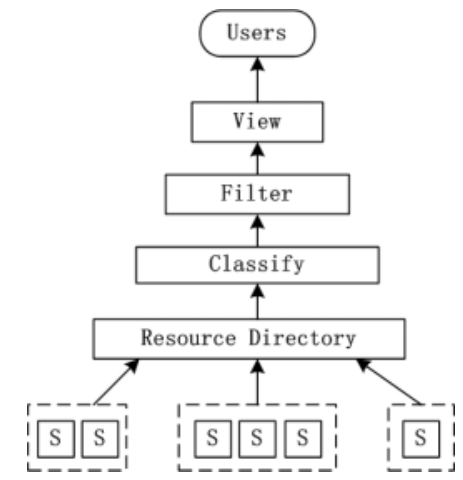

Fig. 7. Layers to provide logical view to users

\subsection{Logical View}

In the agent model, software resources are arranged as a logical information tree from users' point of view. As showed in Fig. 7, in our agent architecture, there are three layers on top of resource directory to provide logical view to users.

Classify layer. Items in directory are classified by their attributes in this layer.

Filter Layer. To give exact information in which users are interested, filter layer is used above the classified layer. Data are filtered according to users' demand in filter layer.

View layer. This layer gives the final logical view to users by some arrangement and process of data from filtered layer. 


\section{Registry of Software Resources}

To initialize the directory, each software entity should be registered as an item in a centralized resource directory that is managed by agent. As is shown in Fig. 8, the registry tool in agent, which is based on Globus API, acts as a LDAP client to store information in the directory.

The realization of register module in our agent is based on the API of MDS (Metacomputing Directory Service). It communicates with the resource directory using LDAP protocol. Users initially register software entities using registry interface. Each entity is processed as an entry, with its information stored in resource directory according to the agent data model (See Sect. 4).

\section{User Discovering Resources}

An XML-based web interface is used to make users' operation easy. Using this interface, Users can query software resources by specifying their demand such as software name or a given software class. As Fig. 9 shows, between web interface and searching engine is a command interpreter. Its function is to convert the data from web page to searching engine, which can recognize and then make appropriate searching policy regarding users' demand. To make sure that every software entity replied to users can be used immediately, when finding results in directory, the agent tests the availability of entities among them, then just returns the available results to users. Unavailable ones are marked and will be tested later.

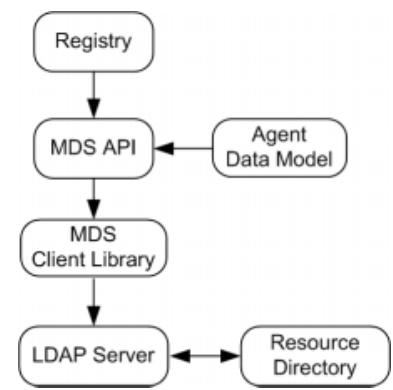

Fig. 8. Implementation architecture of registry tool

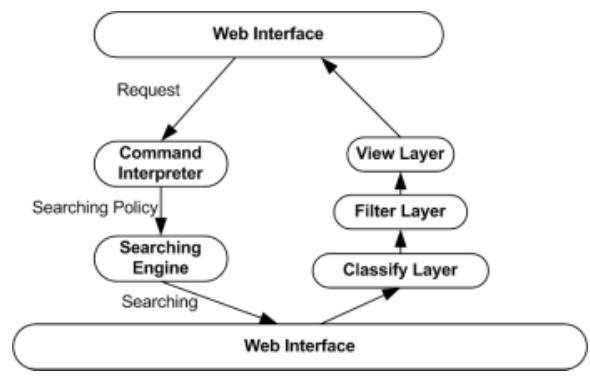

Fig. 9. A command interpreter converts data from web interface to searching engine

\section{Trace of OGSA Proposal}

Building on both Grid and Web services technologies, the Open Grid Services Architecture (OGSA) defines mechanisms for creating, managing, and exchanging information among entities called Grid services. Succinctly, a Grid service is a Web 
service that conforms to a set of conventions (interfaces and behaviors) that define how a client interacts with a Grid service [9].

We have implemented our agent model based on Globus Toolkit 2.0. To take advantage of OGSA, we are now trying to build a service to make distributed software resources sharing. The result is to provide software libraries, which is transparent to users, in grid environment.

\section{Related Work}

A famous related work is Universal Description, Discovery and Integration (UDDI) protocol. It is one of the major building blocks required for successful Web services. UDDI creates a standard interoperable platform that enables companies and applications to quickly, easily, and dynamically find and use Web services over the Internet. UDDI also allows operational registries to be maintained for different purposes in different contexts. UDDI is a cross-industry effort driven by major platform and software providers, as well as marketplace operators and e-business leaders within the OASIS standards consortium [18].

Because of some similarity between UDDI and our agent model, it's necessary to present the difference of them. In brief, the main function of UDDI is to provide a "meta service" for locating web services by enabling robust queries against rich metadata. The purpose of our agent model is to manage a kind of specific resource software resource in grid environment.

\section{Conclusions and Future Work}

We have presented an agent model for sharing distributed software resources in grid computations. It not only simplifies the operation of user, but also provides a uniform interface to users. User can search the resources he needs conveniently and efficiently and get some information about them. In the future, we expect to use the following technologies:

Automatically identify installed software. To avoid registering manually, we would have agents scan file systems to automatically identify installed software.

Multiple agent servers (or backup servers). To realize fault-tolerant operation for user, we can build multiple servers to store information cooperatively. The redundant directories will take over the job when original directories fail due to some faults such as network partition.

Monitoring tool and allocation mechanism. Resources in grid environment can be redundant. If some kind of software resource exists in more than one host, agent should allocate an appropriate lone to user. The resources state such as using rate can be obtained through monitoring tool. Then agent may generate an optimized strategy. The benefit of such mechanism is balancing the load of hosts. 


\section{References}

1. K. Czajkowski, S. Fitzgerald, I. Foster, C. Kesselman: Grid Information Services for Distributed Resources Sharing. Proc. 10th IEEE International Symposium on HighPerformance Distributed Computing (HPDC-10), IEEE Press (2001)

2. S. Fitzgerald, I. Foster, C. Kesselman, G.V. Laszewski, W. Smith, S. Tuecke: A Directory Service for Configuring High-Performance Distributed Computations. Proc. 6th IEEE Symp. on High Performance Distributed Computing

3. G.V. Laszewski, I. Foster: Usage of LDAP in Globus. http://www.globus.org (2002)

4. I. Foster, C. Kesselman, S. Tuecke: The Anatomy of the Grid Enabling Scalable Virtual Organizations. Intl J. Supercomputer Applications (2001)

5. I. Foster, C. Kesselman, The grid: Blueprint for a Future Computing Infrastructure, Morgan-Kaufmann (1999)

6. OpenLDAP 2.1 Administrator's Guide. http://www.openldap.org (2002)

7. MDS 2.2 User's Guide. http://www.globus.org

8. MDS 2.2: Creating a Hierarchical GIIS. http://www.globus.org

9. S. Tuecke, K. Czajkowski, I. Foster, J. Frey, S. Graham, C. Kesselman: Grid Service Specification Draft 4 (10/4/2002). http://www.gridforum.org/ogsi-wg

10. I. Foster, C. Kesselman: Computational Grids. http://www.globus.org

11. Globus: A Metacomputing Infrastructure Toolkit. http://www.globus.org

12. Extensible Markup Language (XML) 1.0 (Second edition) W3C Recommendation. http://www.w3.org/TR/REC-xml

13. M. Livny: Matchmaking: Distributed Resource Management for High Throughput Computing. Proc. 7th IEEE Symp. on High Performance Distributed Computing (1998)

14. J. Rosenberg, H. Schulzrinne, B. Suter: Wide Area Network Service Location. IETF Internet Draft (1997)

15. S. Gullapalli, K. Czajkowski, C. Kesselman, S. Fitzgerald: The Grid Notification Framework. Grid Forum Working Draft GWD-GIS-019. http://www.gridforum.org

16. T.A. Howes, M. Smith: A Scalable Deployable Directory Service Framework for the Internet. Technical report, Center for Information Technology Integration, University of Michigan (1995)

17. R. L. Ribler, J. S. Vetter, H. Simitci, D. A. Reed: Autopilot: Adaptive Control of Distributed Applications. Proc. 7th IEEE Symp. on High Performance Distributed Computing (1998)

18. http://www.uddi.org 